\title{
TTR
}

Traduction, terminologie, re?daction

\section{Mallarmé traducteur, ou le contresens heuristique}

\section{Pierre Godin}

Volume 2, numéro 2, 2e semestre 1989

L'erreur en traduction

URI : https://id.erudit.org/iderudit/037051ar

DOI : https://doi.org/10.7202/037051ar

Aller au sommaire du numéro

Éditeur(s)

Association canadienne de traductologie

ISSN

0835-8443 (imprimé)

1708-2188 (numérique)

Découvrir la revue

Citer cet article

Godin, P. (1989). Mallarmé traducteur, ou le contresens heuristique. TTR, 2(2),

141-151. https://doi.org/10.7202/037051ar d'utilisation que vous pouvez consulter en ligne.

https://apropos.erudit.org/fr/usagers/politique-dutilisation/ 


\section{Mallarmé traducteur, ou le contresens heuristique}

\section{Pierre Gobin}

L'œuvre poétique de Mallarmé est extrêmement condensée. Pourtant, l'ensemble de sa production est relativement volumineux, si l'on y inclut, outre les vers et les textes théoriques, les articles de la Dernière Mode et d'autres revues, les ouvrages scolaires (les Mots Anglais et les Thèmes Anglais) et les traductions. Certes, Mallarmé, dans une lettre célèbre à Verlaine, présente la plupart de ces derniers écrits comme purement alimentaires ( $j$ 'ai dû faire dans des moments de gêne ou pour acheter de ruineux canots, des besognes propres, et voilà tout (Dieux Antiques, Mots Anglais) dont il sied de ne pas parler "). Mais ces "besognes propres" constituent un lien précieux entre les tâches quotidiennes d'enseignement et la quête poétique du livre par excellence, entre le contingent et l'absolu. Elles contribuent à éclairer, en les définissant à des fins de vulgarisation (très relative) les principes théoriques de la recherche de Mallarmé ; elles donnent aussi des exemples concrets de l'exercice du métier d'écrire : l'artisan du langage et l'artiste du verbe ne sont pas opposés mais complémentaires.

Je ne saurais ici examiner en détail les théories linguistiques de Mallarmé et notamment l'évolution de son cratylisme'. Il convient

1. La présentation la plus équilibrée et la plus commode demeure celle de Jacques Michon, Mallarmé et les Mots Anglais, 1978, P.U. Montréal, qui fait le point des travaux antérieurs, de Delfel (1951: "Mallarmé est un Platon qui aurait pris le Cratyle tout à fait aux sérieux ») à Genette (1972 : "Le cratylisme primaire " du héros de Platon est remplacé par le "cratylisme secondaire "du poète ") et établit l'éventail des réactions de Mallarmé. "En poésie, écrit-il, Mallarmé est un Cratyle heureux, dans les Mots Anglais, devant les beautés et les défauts de la langue, il est partagé entre des sentiments contradictoires. » (p. 14) J'ajouterais que dans les traductions le poète joue sur l'ouverture et le repliement de cet éventail. 
toutefois de rappeler brièvement certains principes sur lesquels il revient à maintes reprises et qui aident à mieux comprendre la place relative des divers écrits dans l'ensemble de l'œuvre, et notamment des traductions.

Deux notes ${ }^{2}$ générales, de caractère méthodologique, rédigées en 1869 et 1894 , et qui encadrent donc l'essentiel de sa production poétique, sont particulièrement révélatrices. La plus ancienne marque un effort pour reprendre, à l'instar de Descartes, une analyse du "procédé même de l'esprit humain" qui jetterait les prolégomènes d'une "Science du Langage":

Arriver de la phrase à la lettre par le mot; en nous servant du Signe ou de l'écriture, qui relie le mot à son sens. La Science n'est donc pas autre chose que la Grammaire, historique et comparée, afin de devenir générale, et la Rhétorique. (DE LA SCIENCE) La Science ayant dans le Langage trouvé une confirmation d'elle-même, doit maintenant devenir une confirmation du Langage. (p. 852)

Cette Science, on le voit, procède essentiellement par analyse au départ ; travaillant sur l'objet culturel, donné par l'écriture et fixé, elle remonte à un élément irréductible. Toutefois elle doit, à partir des principes ainsi établis (la lettre, la grammaire générale), s'orienter vers une pratique (combinatoire rhétorique, exercice bien fondé du langage). Par ailleurs, les éléments phonétiques sont entièrement scotomisés (ou du moins pris en charge par la trace écrite, non en tant que sons, mais en tant que lettres). Enfin la recherche des fondements de la science renvoie à un logos premier, qui, s'il n'est pas historiquement primitif ou théologiquement initial, conditionne et légitimise la pratique actuelle ou future : ce qui «doit maintenant devenir» s'exerce à partir de ce qui a été "trouvé ".

Cette position est amplifiée, en termes idéalistes et à grands renforts de capitales, par le jeune poète dans les termes suivants :

Le Langage est le développement du Verbe, son Idée, dans l'Être, le temps devenu son mode : cela à travers les phases de l'Idée et du Temps en l'Être, c'est-à-dire selon la Vie et l'Esprit. D'où les deux manifestations du Langage, la Parole et l'Écriture, destinées (en nous arrêtant à la donnée du Langage) à se réunir toutes les deux en l'Idée du Verbe : la Parole, en créant les analogies des choses par les analogies des sons - L'Écriture en marquant les gestes de l'Idée se manifestant par la parole, et leur offrant leur réflexion, de façon à les parfaire, dans le présent (par la lecture) et à les conserver à l'avenir comme annales de l'effort successif de la parole et de sa filiation...

2. Publiées dans l'édition de la Pléiade sous la rubrique proses diverses, respectivement aux pages 851-854, et 854-856, immédiatement avant l'Avant-dire au Traité de Verbe de Ghil. 
Les rapports mutuels de «la Parole» et de «l'Écriture», et la relation spécifique de chacune de ces manifestations du "Langage", l'une au son, l'autre au geste (dont Mallarmé rappelle plus loin l'insertion physique et physiologique) renvoient ainsi à un rapport des mots et des choses. Si ce rapport inhérent doit être «créé" (par l'écriture, à la fois invention et arabesque, et par la lecture, à la fois déchiffrement et modulation), il est nécessaire et originel. Mallarmé adopte donc une position néo-cratylique ${ }^{3}$. On voit les implications de cette position pour un traducteur : non seulement l'élément référentiel, l'élément iconique et l'élément expressif sont unifiés, mais les différentes langues du monde sont étroitement associées ${ }^{4}$.

La deuxième note méthodologique, beaucoup plus tardive (elle date de 1895, trois ans à peine avant la mort du prince des poètes), est entièrement dépourvue de capitales, et d'entités hypostasiées. Elle est aussi plus concrète, s'appuyant sur une étude spécifique de certains signes (signes actifs et phonétiquement perçus, et «signes éteints (je suppose l's du pluriel)»), en fonction de la poétique. Mallarmé y livre des secrets professionnels ( «je demande pardon de mettre à nu les vieux ressorts sacrés"), spécule sur l'avenir de la rime et du vers, et propose des directions de recherches. Surtout il marque clairement la distinction entre la langue naturelle (vocabulaire de tous, syntaxe héritée, "mots de la tribu» en somme) et le langage poétique qu'il s'agit de créer :

Un vocabulaire appartient en commun, cela seul ${ }^{5}$ au poète et à tous, de qui l'œuvre, je m'incline, est de le ramener perpétuellement à la

3. On connaît les questions qui forment le fond du débat dans le Cratyle de Platon, à savoir "s'il existe une rectitude originelle de dénomination, appartenant de nature à chaque réalité " et "si cette rectitude existe originellement pour Grecs et Barbares et la même pour tous indistinctement " (trad. Robin, éd. de la Pléiade, Section 394). Bien entendu il conviendrait de situer tout ce problème dans le contexte établi par G. Genette.

4. Cette position, qui n'est pas rare chez les savants du XIX ${ }^{e}$ siècle (surtout à partir des travaux linguistiques de Burnouf, des recherches de Max Miller sur les mythes, des hypothèses sémantiques de Bréal) est particulièrement séduisante pour Mallarmé poète. Non seulement "le monde existe pour aboutir à un livre ", mais la lettre originelle précède l'existence du monde. Nous retrouvons le " au commencement était le verbe", sans toutefois que la distinction "le verbe était en Dieu " soit tenue pour pertinente. Il convient cependant d'insister sur le fait que le champ linguistique considéré est surtout indo-européen, en dépit de l'intérêt que Mallarmé a manifesté pour les "langues sémitiques " (lettre de 1870 citée par Michon, p. 127), auxquelles il espérait toutefois, ce qui me semble symptomatique, arriver "par le Zend".

5. On notera que la syntaxe n'est pas explicitement mentionnée ici ; plus loin cependant, Mallarmé oppose "l'armature de la langue ", et la " (philosophie)" en dehors de toute grammaire. $S$ 'il a entrepris dès 1872 la rédaction des Thèmes anglais, avant donc les Mots Anglais, "il faudra attendre encore trois ans avant d'entendre parler de la Petite Philologie ". Cf. sur cette question l'ouvrage de Jacques Michon, p. 39 notamment. 
signification courante, comme se conserve un sol national; dites, le dictionnaire me suffirait: soit, trempez-le de vie, que je devrai en exprimer pour employer les termes en leur sens virtuel.

La recherche dans les arcanes, pour la valeur «purement hiéroglyphique de la parole ou du grimoire ", ne doit plus faire abstraction de la foule ; il s'agit de «subir l'épreuve orale ou d'affronter la diction comme un mode de présentation extérieur" aussi bien que d'explorer «au delà du silence »: le respect envers le passé doit être exempt de toute idolâtrie - le poète est ironique envers "certaines piétés que nous eûmes Parnassiens... qui peuvent sentir leur séminariste, clerc" - et seulement mieux assurer les «libérations ». Dans cet espèce de testament théorique, qui jette un regard rétrospectif sur «l'illumination littéraire principale (...) des vingt années précédentes", le maitre envisage "l'avenir qui n'est jamais que l'éclat de ce qui eût dû se produire antérieurement ou près de l'origine ». Il n'adopte plus un ton d'assurance prophétique ou de didactisme mais livre une spéculation dépourvue d'illusions ( non que cet éclair se soit promulgué simplement pour la broderie de son feu et par dandysme sauf à se retirer en soi"); sans renoncer à la conception d'unité fulgurante du logos originel, il la présente comme une vision chère plutôt que comme une hypothèse : cela « eût dû̉» se produire et non «a dû ».

La distribution hiérarchisable de l'ensemble de la production de Mallarmé se retrouve dans ses traductions, qui vont de simples exercices didactiques, en passant par des travaux de librairie assez hâtifs, à des entreprises longtemps mûries, et révisées avec amour. Mais à chaque niveau des traductions l'on peut déceler la mise en pratique des postulats linguistiques sous-jacents aux recherches poétiques. Mieux, puisque les Thèmes anglais postulent l'utilisation ou du moins la lecture des Mots anglais, on y rencontre l'application de propositions énoncées dans l'étude sur le vocabulaire. Cette étude en effet s'appuie à la fois sur la constatation de parentés historiques entre les langues anglaise et française, et, dans "l'élément anglo-saxon» - par une aptitude développée en termes quasi tainiens par la conjonction d'un lieu et d'un peuple - de rapports intimes entre les mots et les choses, qui, à la limite, débouchent sur une hypothèse concernant les universaux linguistiques. Si les procédés d'allitération sont tenus pour « inhérent(s) au génie septentrional», ils n'en représentent pas moins un

effort magistral de l'imagination désireuse, non seulement de se satisfaire par le symbole éclatant dans les spectacles du monde, mais d'établir un lien entre ceux-ci et la parole chargée de les exprimer, (qui) touche à l'un des mystères sacrés ou périlleux du Langage.

Leur étude présente donc les prolégomènes à une analyse future que l'on pourra conduire «avec prudence" "seulement le jour où la science, possédant le vaste répertoire des idiomes jamais parlés sur la terre, écrira l'histoire des lettres de l'alphabet à travers tous les âges, 
et quelle était presque leur absolue signification, tantôt devinée, tantôt méconnue par les hommes, créateurs de mots».

Dans les classifications qu'il suggère, Mallarmé regroupe les "mots régulateurs", non suivant «l'ordre du Dictionnaire» mais par des rapprochements phoniques ou graphiques, établis «à cause des rapports entre la signification totale de la lettre, plutôt que par un emprunt fait à l'appareil scientifique " 6 . Ainsi,

B... s'appuie, au commencement de chacun des mots, sur toutes les voyelles, peu d'entre les diphtongues, et les seules consonnes 1 et $r$ : cela pour causer des sens, divers et cependant liés secrètement tous de production ou d'enfantement, de fécondité, d'amplitude, de bouffissure et de courbure, de vantardise ; puis de masse ou d'ébullition, et quelquefois de bonté et de bénédiction...(p. 929) F... indique de soi une étreinte forte et fixe (p. 935) G... d'abord une aspiration simple, vers un point où va l'esprit... (p. 938)

ou encore,

$\mathrm{T}$, combiné avec h, pour donner un son absolument nouveau pour nous et pour les latins mais connu de grec, le th fort ou doux, acquiert en anglais le sens le plus net : celui d'objectivité chose et pensée, toi, enfin tous les démonstratifs et l'article commencent ainsi... Tr ne differe pas sensiblement, si ce n'est une fois, en transportant la notion de stabilité dans le sens moral... (p. 953)

Dans les Thèmes Anglais, le plan primitif, inspiré de considérations pédagogiques (rappel des règles de grammaires, regroupement des exercices en fonction de ces règles, «en autant de points capitaux qu'il y a de classes, de cent à cent cinq, dans l'année scolaire») se trouve curieusement infléchi par des postulats lexicologiques, et par un souci d'exploiter le « fonds national » de la langue étudiée. En effet, les thèmes sont eux-mêmes des traductions en français d'expressions consacrées ou de proverbes anglais parfois archaïques ("go fiddle for shives among old wives", XVI; 6) souvent élidés au point que l'expression de surface ne correspond pas à l'ossature syntactique

No secret but between two (XXXIII; 8)

Welcome death, quoth the rat, when the trap fell (LXXXV; 3),

et presque toujours appuyés sur des rimes intérieures ou des allitérations. Il s'ensuit que les phrases françaises apparaissent parfois gauches. Mais, chose plus remarquable, à l'occasion elles contiennent des contre-

6. Ce qui n'exclut pas des références aux racines du sanscrit et des rapprochements avec les autres langues - mais Grimm par exemple n'est pas cité à propos des mutations consonantiques, non plus que Bopp ou Burnouf sur les rapports entre les langues. On notera en outre que les associations ne sont pas seulement établies dans le domaine anglo-saxon ( $\mathrm{B}$ est associé à bonté, $\mathrm{F}$ à force) ni même dans le contexte proprement linguistique, puisque l'horizon est défini par une vertu ou « un point où va l'esprit ». 
sens caractérisés : ainsi «la malpropreté est la mère de la pauvreté " (ex. III ; phrase 3) est censée se traduire par «Sloth is the mother of poverty " qui naturellement renvoie à la paresse. L'erreur de Mallarmé ne peut guère s'expliquer que par un souci d'assonnance, ou de "rime intérieure » en français ; dans d'assez nombreux cas, un calque pur et simple rend la phrase à peu près incompréhensible: "Toute chose à une fin, et un pudding a deux bouts" $(X ; 10)$ ne s'explique que si l'on pense que pudding veut aussi dire boudin (il se peut qu'ici Mallarmé ait reculé devant la cacophonie de «boudin à deux bouts!»).

En général, malgré les notes qui suivent chaque exercice, et l'application de règles qui mettent en évidence les différences de structure des deux langues, l'énoncé français (second) suit de près les linéaments de l'énoncé anglais (prétendument second, mais premier en fait). L'observation de Nida selon laquelle le traducteur tend à porter un respect exagéré aux structures de la langue de départ, surtout si ce n'est pas la sienne propre, se trouve ici confirmée. Elle le sera bien davantage dans les traductions par Mallarmé de textes poétiques «sacrés». Devant les traductions de proses, toutefois, le poète adopte une attitude plus indépendante. L'ouvrage intitulé «les Dieux Antiques" est même en fait plutôt une adaptation de la grande Mythologie des Peuples Aryens du révérend George Cox qu'une véritable traduction; Mallarmé refond complètement le texte, regroupant les mythes de façon analytique, éliminant les longues digressions touffues et les parenthèses, condensant les remarques générales sur l'histoire des religions ou l'histoire des langues ${ }^{7}$, et au besoin ajoutant des sections de son propre cru. Le conte de $\mathrm{M}^{\mathrm{me}}$ Elphinstone Hope, The Star of the Fairies, n'appelle guère de commentaires: "Mallarmé lui a rendu plus que justice en le traduisant ${ }^{8}$. Un examen du texte français cependant ne laisse guère oublier qu'il ne s'agit pas d'un original : la fréquence inusitée des adjectifs précédant les noms ( « mignonne dame »; « l'importune vieille »; une «si étincelante personne»; « un mignon tube poli»), les calques de tournures idiomatiques («les créatures ses pareilles»;

7. Il tient compte aussi des travaux ultérieurs. Rédigé en 1868 et publié en 1870 . l'ouvrage de Cox était déjà un peu daté quand Mallarmé l'adapta en 1879-80. Par exemple, les doutes sur l'historicité de Troie avaient été rendus caducs par les fouilles de Schliemann (dont les résultats ont été publiés à partir de 1870). Contrairement à ce qu'indique la note de l'édition de la Pléiade, c'est la Grande Mythologie et non les "Contes Mythologiques» de 1866-67 qui servait de base à Mallarmé.

8. Voir note de l'édition de la Pléiade. L'original, édité en anglais en France, ne semble pas avoir connu un grand succès. Mallarmé ne consacra que quelques semaines à cette besogne, alors qu'il avait longuement pratiqué l'ouvrage de Cox, dont certaines idées sur les langues rencontraient les siennes propres, malgré la tournure plus théologique de l'érudit anglais, qui cherchait une unité idéale vers la nature révélée. 
"c'est trop joli"; «elle ferait son étude du plaisir d'autrui », ou de structures syntactiques («murmurant tout comme elle allait»), et même certains contresens extraordinaires ("le sentier des Lys de la Vallée ", là où il s'agit naturellement de muguets, «distillez cette eau sur les paupières des géants " pour "versez en quelques gouttes") renvoient évidemment à l'anglais. Ces contresens, qui résultent de confusions entre des faux-amis, ont toutefois une saveur particulière ; évoquant titre balzacien ou sortilège shakespearien, ils transmuent une poursuite laborieuse en progression mythique, en enquête sacrée, ouvrant des perspectives proprement poétiques ${ }^{9}$.

C'est dans les traductions poétiques que l'on peut déceler le mieux ce que j'appelle contresens heuristiques, portant Mallarmé audelà des embûches immédiates vers l'intuition d'une unité plus profonde ${ }^{10}$; ces contresens seraient scientifiquement justifiables en théorie, puisqu'une transformation poussée pour remonter vers un algorithme syntactique ou vers un formant lexical tendait à rencontrer des structures voisines de celles d'une autre langue à un même niveau ${ }^{11}$; esthétiquement, ils mettent une tache dans le texte d'arrivée, qui contribuent à lui donner une « couleur " permettant de percevoir quelque chose de l'original, et ne constituent ainsi qu'un cas extrême (et révélateur) d'une technique de reconstitution créatrice ${ }^{12}$. Si Mallarmé ne tente pas de rendre les effets métriques de l'original et contribue ainsi à renforcer l'idée que beaucoup de traducteurs en d'autres langues

9. Les Contes indiens, "arrangés et récrits " par Mallarmé à partir de textes de Mary Summer déjà traduits, et le Ten $O^{\prime}$ 'clock de Whistler, dont le texte français résulte d'une collaboration avec Viélé-Griffin, poète parfaitement bilingue, ne relèvent pas de notre examen.

10. J'examine ici surtout les traductions de Poe. Pour les poèmes de Tennyson, la version de Mariana est assez plate ; celle de Godiva, inédite jusqu'en 1970, et dont un brouillon (1874 ?) et une transcription incomplète (1880 ?) ont été publiés dans Documents Stéphane Mallarmé II (Paris, Nizet), avec une présentation de Carl Paul Barbier, appellerait une étude particulière - on y voit à l'cuvre un traducteur scrupuleux et fin - mais elle ne présente pas d'anomalies révélatrices.

11. Cf. Nida, Eugene, "Science of Translation», Language, vol. 45, no. 3, 1969 : "It would seem that ultimately the deepest structural level involves simply a pool of semantic universals " (p. 487), et plus loin (p. 489), "One could conceive of languages having widely differing surface structures but with kernel structures converging toward a center. "

12. Mounin consacre un excellent développement à Mallarmé et ses «calques » dans les Belles Infidèles (Paris, Cahiers du sud, 1953), pp. 141-142, «(sa) traduction (de Poe) vise à créer le sentiment de la syntaxe anglaise en français.. " 
se font de "l'approche française" ${ }^{13}$, il s'efforce d'en calquer les structures de surface. Certes, les traductions comportent des maladresses inexcusables, et l'on pourrait ajouter des pages de gloses à celles de Viélé-Griffin ou d'Émilie Noulet ${ }^{14}$. Erreurs de lectures (ainsi rhyme est rendu par «rythme» dans la Romance) ou de relecture (elf devient «elle» dans Silence, "le cœur étoilé " traduit the starry choir dans Israfel, sans doute par confusion avec les fibres du cour de l'ange musicien, et dans le même poème "is the sunshine of ours" devient "est le sommeil de la nôtre ") ; coquilles caractérisées ; ruptures de ton ou de rythme («allant, elle, d'accord», pour «keeping time» dans les Cloches) ; chevilles et platitudes (attend the spell, dans Israfel devenant "se prennent au charme"); omissions; faux sens (dirge rendu par "psalmodie" dans Léonore; wanderer par "promeneur" dans Terre de songe) et même contresens manifestes ("quiétude» pour darkness, «en quelque sorte plus fort" pour somewhat louder dans le Corbeau) et j'en passe. Mais certaines "paraphrases» ou inexactitudes au dire de Viélé-Griffin représentent d'heureuses équivalences phoniques : dans le Corbeau «Et de la soie l'incertain et triste bruissement " pour And the silken and uncertain rustling, à la strophe 3 ; et plus loin, à la strophe 7, «Maints enjouements et agitations d'aile », pour Many a flirt and flutter; des erreurs littérales ouvrent une nouvelle dimension poétique ${ }^{15}$ : the lolling lily de Terre de Songe est certes plus exactement

13. Cf. par exemple l'article de V.V. Levik " La traduction et la création littéraires ", dans The Nature of Translation (James S. Holmes ed., The Hague, Mouton, 1970), p. 166: «Il est de tradition chez nos collègues français de traduire les vers en prose... mais les traducteurs ne se nuisent-ils pas à eux-mêmes en anéantissant, dans l'embryon même, la possibilité d'enrichir le trésor de la poésie française... " Pour une réfutation de cette idée d'une "tradition" française, également exprimée par Max Beerbolhm entre autres, voir Paul Selver, The Art of Translating Poetry, London, John Baker, 1966, pp. 15-20

14. Voir éd. de la Pléiade, notes pp. 1529-1535. Les deux critiques cités n'ont d'ailleurs examiné que certains poèmes de Poe: le Corbeau (Viélé-Griffin ; Noulet) ; Ulalume (V.-G.) ; Léonore (V.-G. ; N.) ; Annabel Lee (V.-G. ; N.) ; la Dormeuse (V.-G.); les Cloches (V.-G.); Israfel (V.-G.); Terre de Songe (V.-G.) ; À Hélène (N.); Pour Annie (N). J'ai relevé des anomalies notoires également dans la Cité en la mer, le Palais hanté, Romance, le Colisée. Madame Noulet établit des comparaisons intéressantes entre les versions de Mallarmé et de Baudelaire, en ce qui concerne le Corbeau.

15. Emilie Noulet s'interroge sur la portée de cette transmutation, et sur la question de savoir en quoi elle peut avoir modifié le style de Mallarmé dans ses œuvres originales : «... Il est possible, écrit-elle, qu'une connaissance suffisante mais encore incomplète de l'anglais et la nécessité de la traduire aient contribué à donner à la langue française une élasticité qu'elle ne connaissait pas, un emploi de mots qu'elle ne se permettait pas, et une diversité qu'elle ne souhaitait pas. " (1'Guvre poétique de Stéphane Mallarmé, Paris, Droz, 1940, p. 172). Cette interprétation me semble valable, bien que l'on doive émettre des réserves sur " la connaissance incomplète " d'un professeur déjà chevronné, ayant publié des ouvrages didactiques sur la langue en question, et travaillé plus de dix ans sur l'œuvre à traduire. Quant à la "nécessité ", les avatars de la publication des Poèmes d'Edgar Poe semblent bien indiquer qu'elle n'était pas pressante. 
rendu par "des nénuphars qui sommeillent», ainsi que le propose Griffin, mais le «des lys inclinés" de Mallarmé est un calque fidèle à la thématique et à la tonalité du poème; that drip all over, dans le même poème, n'est pas trahi par " qui perlent au-dessus "; dans Lenore, vous et moi traduirions lowly par «humblement », comme le fait Griffin mais le «profondément» de Mallarmé amplifie les échos de ce poème funèbre; dans Ulalume, crisp and sere n'a jamais signifié «crispés et mornes ", mais ce calque et ce tic d'écriture contribuent à l'illusion lyrique (pathetic fallacy) et renforcent la réaction du poète à l'atmosphère de «terre gaste» qui s'exhale du paysage.

Dans la Cité en la Mer, sculptured ivy est transposé en «ifs sculptés", conforme aux sons et aux quantités, et surtout, pour qui connaît les cimetières méditerranéens, plus évocateur que le lierre; dans le même poème à light out from the lurid sea Mallarmé substitue « une clarté sortie de la mer livide», où le faux sens, bien que laissant échapper les connotations sinistres, renforce la relation du paysage à un état d'âme, rend compte étymologiquement de la qualité de la clarté, et établit une quasi-équivalence phonique.

Romance, pour le vers 17 , nous propose un sérieux glissement sémantique : Its down upon my spirit flings n'est pas exactement rendu par «étend sa plume sur mon esprit »; la plume, si elle peut servir à remplir un édredon comme the down, évoque surtout pour un Français la plume d'oie du greffier, le calame de l'ange qui tient le registre, voire les rémiges de l'aile de cet ange. Mallarmé suggère un icte net, et un ascendant intellectuel ou spirituel, alors que Poe donne une image sensible, assortie d'un oxymoron où la mollesse feutrée du duvet est niée par la dynamique violence de la projection (flings). Pourtant, le traducteur atténue la netteté de "plume» grâce au verbe «étend", et restaure en partie du moins l'effet troublant de l'oxymoron.

Dans la Dormeuse, pour The pale-sheeted ghosts go by, "pendant qu'errent les fantômes aux plis obscurs » laisse perdre les effets sonores (peu heureux d'ailleurs, et inappropriés ("fantômes à go-go!»)) et offre un terme contrasté pour rendre pale-sheeted. Mais "aux linceuls pâles » par exemple donnerait dans l'épithète de nature, la convention de certaine «diction» du XVIII ${ }^{e}$ siècle, à laquelle l'original n'échappe pas tout à fait, alors que «aux plis obscurs » renforce l'impression de mystère, tout en évoquant des souvenirs classiques. Il y a en somme substitution de figures, et je crois que le texte de Mallarmé est à bien des égards supérieur ici à celui de Poe, surtout si l'on pense à la rupture de ton de "go by".

Dans le Colisée enfin, si «changé" pour humble à la première strophe, «s'inclinait » pour lolled à la troisième me semblent des erreurs injustifiables, et, tout à la fin «en plus que de la gloire», pour a robe of more than glory une espèce de galimatias, assorti d'un solécisme, la traduction de pallid stones par «passives pierres" me semble rendre 
compte des effets sonores, et renforcer l'assertion du début du vers "nous ne sommes pas impuissantes», version exacte de we are not impotent.

Mallarmé, traducteur de poésie, ne choisit pas de suivre son modèle dans la recherche d'effets rythmiques ou euphoniques. Il s'en excuse d'ailleurs dans les scolies, et répond à l'avance à l'objection de Viélé-Griffin concernant The Bells ("la raison d'être de ce poème... étant dans son rythme aux rimes répétées, aux allitérations incessantes, il reste intraduit et intraduisible »), en renvoyant à «l'essai d'imitation » d'Émile Blémont qui a pu, «s'éloignant du calque strict habituel de notre version, transposer d'une langue à l'autre tels timbres jumeaux ". Mais il a réussi, malgré son choix du calque, et parfois grâce à ce choix, à offrir un texte susceptible de stimuler l'imagination des poètes français, et de servir la gloire de Poe, devenant prophète hors de son pays. Il a, en dépit de ses maladresses et parfois en les transcendant, offert en prose un énoncé poétique fidèle, et en même temps original, monument que vient couronner le sonnet du Tombeau, défi au temps dans sa thématique et dans son expression.

Pratiquement à chaque fois qu'il opère une transposition ou effectue un glissement/un calque/ «transfer ", Mallarmé joue sur les similarités des structures de surface. Cela le conduit à privilégier les termes d'apparence semblable, et les tours syntactiques identiques, ce que Mounin appelle la traduction à travers des verres colorés. II lui arrive de tomber dans la «traduisite» («translationese») ou de proposer des faux-amis. Il opère même des assimilations hasardeuses entre termes de la langue de départ qui le conduisent à des contresens caractérisés.

Toutefois, lorsqu'il effectue de tels glissements, c'est systématiquement dans le sens d'une identification phonique ou syntactique capable de stimuler la recherche d'un niveau d'expression plus général et de remonter par une transformation («back transform») dans la direction d'universaux linguistiques, philologiquement fondés ou résultant d'une certaine perception poétique de l'unité.

Les traductions de Mallarmé offrent donc de beaux exemples du danger et de la grandeur d'un effort d'analyse excessif, allant de pair avec ce que Nida décrit comme un respect quasi religieux du texte de départ. Ce respect, conscient dans le cas des poèmes de Poe, dont la lecture détermina pour une large part la vocation d'angliciste de Mallarmé, s'étend aux autres textes anglais considérés, non sur le plan référentiel (Mallarmé n'hésite jamais à couper, «éditer ", transposer des récits ou des exposés) mais sur le plan poétique du langage. Il amène à poétiser des éléments qui relèveraient du métalangage, et de la langue comme corpus. 
Mais, de façon complémentaire, Mallarmé, créateur en sa propre langue de structures fortement synthétiques, même si elles se présentent comme diluées, concentre souvent sur le texte d'arrivée des effets épars initialement. Dans les textes didactiques ou les ouvrages de vulgarisation, il s'agit d'une intervention délibérée (constitution d'une taxinomie ; établissement d'une progression méthodique ; recomposition en clair d'un discours diffus ; promotion au statut littéraire de productions quasi littéraires). Dans les traductions de poètes, il n'y a pratiquement pas de modification de l'économie de l'original. Mais si le mot à mot scrupuleux, le "calque " de surface, met en évidence, outre le style personnel du poème "source", les caractères que le traducteur attribue à la langue de départ, il révèle aussi parfois, dans les rapprochements hasardeux et les tours inusités, le fonctionnement de la faculté poétique propre de traducteur. Il ne renvoie pas au cliché, à l'expression usée des "mots de la tribu ", mais à un sens plus "pur", même s'il propose une substitution. Pour reprendre la métaphore traditionnelle, Mallarmé traducteur n'offre pas des pièces de billon courantes, en échange d'une monnaie d'or, mais frappe des médailles, marquées de son propre coin, même lorsque l'effigie est reconnaissable. Sa traduction est poétique, non parce qu'elle rend les rythmes ou les effets prosodiques de l'original, ou parce qu'elle se présente comme un poème, mais parce qu'elle incite à l'exercice original d'une lecture poétique ${ }^{16}$. Là même où elle est infidèle au produit (c'est-à-dire à la chose qu'est l'œuvre de départ), elle est fidèle à la production (lecture ou écriture), "à l'effet qu'elle produit " comme à l'idée qui la produit, à la démarche créatrice. En ce sens, elle représente une étape vers la recherche personnelle du Livre à venir.

Queen's University

16. Selver note avec raison que c'est inverser abusivement les termes que de prétendre que seul un poète saura traduire un poète : un traducteur digne de ce nom devient poète s'il réussit à rendre l'original poétique (p. 47). En ce qui concerne Mallarmé, la démarche est différente, et suppose une double remontée vers une "source» absolue. 